\title{
Surveillance strategies after definitive therapy of invasive bladder
}

\section{cancer}

\author{
Ilias Cagiannos, MD, FRCSC; Christopher Morash, MD, FRCSC
}

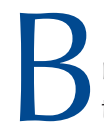

Abstract

Following definitive therapy for muscle invasive bladder cancer, patients remain at risk for local and distant recurrence. Additionally, recurrences can result from formation of new tumours elsewhere in the urinary tract. We review patterns of recurrence and the prognosis associated with recurrence. Optimal surveillance strategies are discussed.

Can Urol Assoc J 2009;3(Supp|4):S237-42 ladder cancer is the second most common urologic malignancy after prostate cancer. ${ }^{1}$ At the time of initial diagnosis, $19 \%$ of patients present with muscleinvasive disease. ${ }^{2}$ In the absence of distant metastatic disease, these patients are most commonly treated with radical cystectomy, pelvic lymphadenectomy and urinary diversion. A select few patients will undergo bladder-preserving protocols. Following treatment, patients can recur with local or metastatic disease as well as with the development of new urothelial tumours in the upper urinary tract or urethra. There is no clear consensus on the optimal schedule of post-treatment surveillance.

\section{Local recurrence}

Local recurrence following cystectomy encompasses pelvic soft-tissue involvement and involves pelvic lymph nodes. However, there is not a uniform definition on the proximal extent of the nodes distinguishing local from distant recurrence. Some authors define local recurrence as involvement of the nodes below the iliac bifurcation, whereas others have extended this definition to include the aortic bifurcation. ${ }^{3,4}$ Possible mechanisms of local pelvic recurrence include incomplete resection of the tumour, resulting in grossly or microscopically positive surgical margins, involved unresected lymph nodes beyond the limits of the lymphadenectomy, and intraoperative spillage of tumour cells. ${ }^{3}$

The frequency at which local recurrence occurs has decreased significantly from the nearly $40 \%$ reported in early cystectomy series. ${ }^{5,6}$ More contemporary series report local recurrence rates of $3.9 \%$ to $29 \%$ (Table 1 ). Factors associated with local recurrence include pathologic tumour stage, presence of tumour in regional lymph nodes, extent of lymphadenectomy and use of perioperative chemotherapy. ${ }^{4}$ Patients with extravesical disease are at much higher risk of local recurrence. Rates of local recurrence were $6 \%$ to $18 \%$ in patients with stage pT1 to pT3a (based on 1992 TNM [tumour, node, metastasis] staging) compared to $51 \%$ for those with stage pT3b in a study by Greven and colleagues. ${ }^{7}$ In a long-term analysis of 1054 patients, Stein and colleagues observed an overall local recurrence rate of $7 \%$ and rates of $6 \%$ and $13 \%$ for those with organconfined and extravesical lymph node negative patients. ${ }^{11}$ Patients with pelvic lymph node involvement are also at increased risk of local recurrence, with reported rates of $24 \%$ to $43 \% .7,8,12,13$ Interestingly, in the study by Stein and colleagues, those with lymph node-positive disease demonstrated only a $13 \%$ local recurrence rate following cystectomy. Patients in this study underwent a meticulous extended lymphadenectomy extending proximal to the aortic bifurcation. The low rate of local recurrence observed in this study suggests a benefit of extended lymphadenectomy in decreasing pelvic recurrences. Other studies have shown improved local control as well as cancer-specific and recurrence-free survival in patients having more extensive lymphadenectomies. ${ }^{14,15}$

Time to development of local recurrence is relatively short, with median time being 9 to 18 months and typically less than 12 months. ${ }^{7,10,11,16,18}$ Westney and colleagues, in a review of their institution's experience with local recurrence over 35 years, reported that although 27 of 33 patients $(82 \%)$ experienced local recurrence within 2 years, the range was from 1 to 72 months. ${ }^{16}$ Most patients presenting with local recurrence are symptomatic. In the study by Westney and colleagues, 25 out of 33 patients (76\%) had symptoms and in 10 patients (30\%) there was a palpable pelvic mass. ${ }^{16}$ In the series by Wishnow and Dmochowski, all 10 patients with local recurrence had symptoms. ${ }^{8}$ The most common symptoms are pelvic, perineal, gluteal or hip pain. Bleeding or discharge from the vagina or urethra as well as lower extremity edema are less common symptoms. Asymptomatic local recurrences are detected with axial imaging, most commonly computed tomography (CT) of the abdomen and pelvis. Locally recurrent lesions may be indistinct on CT and some have suggested the superiority of magnetic reso- 


\begin{tabular}{|c|c|c|}
\hline Publication & Years of study & Local recurrences (\%) \\
\hline Greven et al. ${ }^{7}$ & 1977-1986 & 13/83 (18) \\
\hline Wishnow and Dmochowski ${ }^{8}$ & 1983-1985 & 10/178 (5.6) \\
\hline Brendler et al. ${ }^{9}$ & $1982-1988$ & 3/76 (3.9) \\
\hline Schoenberg et al..$^{10}$ & 1982-1989 & $5 / 101(5)$ \\
\hline Stein et al. ${ }^{11}$ & 1971-1997 & 77/1054 (7) \\
\hline Volkmer et al. ${ }^{18}$ & 1986-2006 & $182 / 1270(14.3)$ \\
\hline Frazier et al. ${ }^{21}$ & $1969-1990$ & $50 / 531(9.4)$ \\
\hline Cheng et a. ${ }^{20}$ & 1980-1984 & 63/218 (29) \\
\hline
\end{tabular}

nance imaging (MRI) in delineation. ${ }^{3}$ The prognosis associated with local recurrence is, unfortunately, poor. Simultaneous systemic metastases occur in $36 \%$ to $64 \%$ of patients. ${ }^{16,17}$ Median survival was 7 months in the review by Westney and colleagues, and 4 months in the study by Greven and colleagues.7,16 Aggressive treatment with chemotherapy, surgery or radiation, either in combination or alone, does little to alter the outcome, although it does provide significant palliation. ${ }^{16}$

\section{Distant recurrence}

Most patients who recur following radical cystectomy do so with metastatic disease. The outcomes of patients undergoing radical cystectomy for the treatment of invasive bladder cancer are summarized for several contemporary series in Table 2. Recurrence-free survival ranges from $61.7 \%$ to $76 \%$ at 5 years and $55.2 \%$ to $73 \%$ at 10 years. Increasing pathologic stage and lymph node-positive disease are generally associated with higher recurrence rates and worse survival. Table 3 shows the outcomes in several cystectomy series stratified by pathologic stage and lymph node status.

In an analysis of patients undergoing cystectomy at the University of Southern California, over a 25 year period, distant recurrences increased progressively from $13 \%$, in those with organ-confined lymph node-negative tumours, to $32 \%$, in those with extravesical lymph node-negative tumours. Distant recurrences occured in $52 \%$ of patients with positive lymph nodes. In the Mayo Clinic experience, 10 -year metastasis-free survival decreased from $82 \%$, in those with organ confined cancers, to $54 \%$, in those with extravesical disease. ${ }^{20}$ Metastasis-free survival was $38 \%$ at 5 years and $21 \%$ at 10 years in patients with positive lymph nodes compared to $81 \%$ and $78 \%$ in those with negative lymph nodes.

Metastatic disease develops relatively quickly, with $80 \%$ to $90 \%$ of distant recurrences identified within 3 years. ${ }^{4}$ In a large series from Ulm, Germany, median time to the development of metastatic disease was 13 months. Sites of recurrence were bone in $13.9 \%$, liver in $10.7 \%$ and lung in $9.9 \%{ }^{18}$ The involvement of other sites, such as brain, peritoneal cavity, skin and vagina, occurs less frequently. ${ }^{4}$ The prognosis of patients with metastatic disease unfortunately remains poor. Combination chemotherapy with methotrexate, vinblastine, doxorubocin and cisplatin (MVAC) or gemcitabine and cisplatin (GC) are the current standards however even with these treatments median survival is 13.8 to 14.8 months. ${ }^{22,23}$ Bajorin and colleagues identified Karnofsky performance status of less than $80 \%$ and the presence of visceral (lung, liver or bone) metastases as prognostic factors predicting outcome following treatment with MVAC. Median survival for patients with 0,1 or 2 risk factors was 33, 13.4 and 9.3 months, respectively. ${ }^{24}$ This data may serve as potential evidence that earlier detection of metastatic disease in the asymptomatic state or with smaller disease burden may result in improved response to treatment.

\section{Urethral recurrence}

Recurrent urothelial carcinoma in the retained urethra following cystectomy has historically been shown to occur in only $6 \%$ to $10 \%$ of patients. ${ }^{25,26}$ Urethral recurrences occur even less frequently in patients undergoing orthotopic as compared to cutaneous urinary diversions. In a large series of 768 men with 397 undergoing orthotopic diversion (median

\begin{tabular}{|c|c|c|c|c|c|c|c|}
\hline \multirow{2}{*}{ Publication } & \multirow{2}{*}{ Years of study } & \multicolumn{2}{|c|}{$\%$ RFS } & \multicolumn{2}{|c|}{$\%$ CSS } & \multicolumn{2}{|c|}{$\%$ OS } \\
\hline & & 5 years & 10 years & 5 years & 10 years & 5 years & 10 years \\
\hline Stein et al. ${ }^{11}$ & 1971-1997 & 68 & 60 & $\mathrm{~N} / \mathrm{A}$ & N/A & 66 & 43 \\
\hline Volkmer et al. ${ }^{18}$ & 1986-2006 & 61.7 & 55.2 & N/A & N/A & N/A & N/A \\
\hline Dalbagni et al. ${ }^{19}$ & 1990-1993 & $\mathrm{N} / \mathrm{A}$ & N/A & 57 & N/A & 45 & N/A \\
\hline Cheng et al. ${ }^{20}$ & 1980-1984 & 76 & 73 & 72 & 67 & 53 & 41 \\
\hline Frazier et al. ${ }^{21}$ & 1969-1990 & N/A & N/A & 59 & 46 & 48 & 33 \\
\hline
\end{tabular}

$\mathrm{RFS}=$ recurrence-free survival; $\mathrm{CSS}=$ cancer-specific survival; $\mathrm{OS}=$ overall survival; $\mathrm{N} / \mathrm{A}=$ not available . 


\begin{tabular}{|c|c|c|c|c|c|c|c|}
\hline \multirow{2}{*}{ Publication } & \multirow{2}{*}{$\begin{array}{l}\text { Pathological } \\
\text { group }\end{array}$} & \multicolumn{2}{|c|}{$\%$ RFS } & \multicolumn{2}{|c|}{$\%$ CSS } & \multicolumn{2}{|c|}{$\%$ OS } \\
\hline & & 5 years & 10 years & 5 years & 10 years & 5 years & 10 years \\
\hline \multirow[t]{6}{*}{ Stein et al. ${ }^{11}$} & Organ-confined & 80 & 77 & $\mathrm{~N} / \mathrm{A}$ & $\mathrm{N} / \mathrm{A}$ & 74 & 54 \\
\hline & $\mathrm{N}-$ & 85 & 82 & $\mathrm{~N} / \mathrm{A}$ & $\mathrm{N} / \mathrm{A}$ & 78 & 56 \\
\hline & $\mathrm{N}+$ & 46 & 44 & $\mathrm{~N} / \mathrm{A}$ & N/A & 45 & 37 \\
\hline & Extravesical & 46 & 44 & $\mathrm{~N} / \mathrm{A}$ & N/A & 37 & 22 \\
\hline & $\mathrm{N}-$ & 58 & 55 & $\mathrm{~N} / \mathrm{A}$ & $\mathrm{N} / \mathrm{A}$ & 47 & 27 \\
\hline & $\mathrm{N}+$ & 30 & 30 & $\mathrm{~N} / \mathrm{A}$ & $\mathrm{N} / \mathrm{A}$ & 25 & 17 \\
\hline \multirow[t]{2}{*}{ Dalbagni et al. ${ }^{19}$} & Organ-confined & $\mathrm{N} / \mathrm{A}$ & $\mathrm{N} / \mathrm{A}$ & $\mathrm{N} / \mathrm{A}$ & $\mathrm{N} / \mathrm{A}$ & $59-64$ & $\mathrm{~N} / \mathrm{A}$ \\
\hline & Extravesical & N/A & N/A & N/A & $\mathrm{N} / \mathrm{A}$ & $26-27$ & $\mathrm{~N} / \mathrm{A}$ \\
\hline \multirow[t]{4}{*}{ Cheng et al. ${ }^{20}$} & Organ-confined & $\mathrm{N} / \mathrm{A}$ & 82 & $\mathrm{~N} / \mathrm{A}$ & 78 & $\mathrm{~N} / \mathrm{A}$ & 53 \\
\hline & Extravesical & $\mathrm{N} / \mathrm{A}$ & 54 & $\mathrm{~N} / \mathrm{A}$ & 47 & $\mathrm{~N} / \mathrm{A}$ & 23 \\
\hline & N- & $\mathrm{N} / \mathrm{A}$ & 78 & $\mathrm{~N} / \mathrm{A}$ & 74 & N/A & 46 \\
\hline & $\mathrm{N}+$ & N/A & 31 & N/A & 21 & N/A & 11 \\
\hline \multirow[t]{2}{*}{ Frazier et al. ${ }^{21}$} & Organ-confined & $\mathrm{N} / \mathrm{A}$ & $\mathrm{N} / \mathrm{A}$ & $64-82$ & $48-71$ & $\mathrm{~N} / \mathrm{A}$ & $\mathrm{N} / \mathrm{A}$ \\
\hline & Extravesical & $\mathrm{N} / \mathrm{A}$ & $\mathrm{N} / \mathrm{A}$ & 39 & 23 & $\mathrm{~N} / \mathrm{A}$ & $\mathrm{N} / \mathrm{A}$ \\
\hline
\end{tabular}

$\mathrm{N}$ - = lymph node-negative; $\mathrm{N}+$ = lymph node-positive; RFS = recurrence-free survival; $\mathrm{CSS}$ = cancer-specific survival; OS = overall survival.

follow-up 10 years) and 371 having cutaneous diversion (median follow-up 19 years), Stein and colleagues sought to determine rates and risk factors for urethral recurrence. ${ }^{27}$ They found a $4 \%$ urethral recurrence rate with orthotopic diversions and $8 \%$ with cutaneous diversions. The only significant predictor of increased recurrence risk was prostatic involvement. If prostatic stromal involvement was present, the 5 -year risk of recurrence rose to $11 \%$ in the orthotopic group and $24 \%$ in the cutaneous diversion group. The authors recommend the intra-operative frozen section from the urethra at the prostatic apex as the only criterion to determine whether a urethrectomy should be performed. This recommendation is supported by several other studies. ${ }^{26-28} \mathrm{~A}$ review of several large published series showed low urethral recurrence rates of $0 \%$ to $2 \%$ in women with a negative intraoperative frozen section of the bladder neck. ${ }^{29}$ Urethral recurrences occur at a median range of 1.5 to 2.2 years, with most occurring before 5 years. ${ }^{26,30-35}$ Late recurrences can occur with some diagnosed up to 20 years after radical cystectomy. ${ }^{26,33}$ Urethral recurrences are often associated with poor outcomes, with one report showing a median survival of 28 months. ${ }^{33}$ Stage at diagnosis was the most important predictor of outcome in that series. Therefore, earlier detection would seem to be important in improving survival outcomes. However, the 2 largest series in the literature found no difference in survival in patients having routine urethral wash cytology compared to those diagnosed based on symptoms. ${ }^{33,36}$ This calls into question the utility of routine urethral wash cytology. Caution must be exercised here, as these series are small and limited by their retrospective methodology. It seems clear that any symptom relative to the retained urethra should be evaluated with endoscopy and wash cytology. Routine scheduled urethral wash cytology may still be reasonable pend- ing more clear direction from the literature regarding impact on survival outcomes.

\section{Upper tract recurrence}

The multifocal nature of urothelial cancer leads to a risk of metachronous upper urinary tract (UUT) recurrence development in patients who have undergone radical cystectomy and urinary diversion for bladder cancer. This risk is relatively low (1\% to $9 \%$ ). ${ }^{37-40}$ This low risk is partly due to the high rate of early mortality from bladder cancer. Upper urinary tract recurrence is also often a delayed event and is the most common site of recurrence after 3 or more years of disease-free survival following cystectomy. ${ }^{37-40}$ The 3 - and 5-year cumulative risks of UUT recurrence has been reported to be $4 \%$ and $7 \%$, respectively. Analysis of this data over time showed that the $4 \%$ to $6 \%$ risk seen at 3 years remained constant for any point in time up to 4 years post-cystectomy. ${ }^{41}$ There are reported cases of UUT recurrence found as long as 9 years following cystectomy. ${ }^{37,38,40-43}$ Most UUT recurrences present with symptoms, such as hematuria or flank pain. Only a minority are picked up with routine surveillance imaging. ${ }^{37-40}$ In a recent review of published series, $58 \%$ to $78 \%$ of UUT recurrences presented with symptoms and $22 \%$ to $42 \%$ were found on surveillance imaging or cytology at a mean of 43 months post-cystectomy. ${ }^{46}$ Urinary cytology detects less than $30 \%$ of UUT recurrences when used for routine post-cystectomy surveillance. ${ }^{39-41,43}$ However, in patients with symptoms, such as hematuria, cytology is positive in $80 \%$ to $100 \%$ of patients with recurrence, thereby making it helpful to confirm the diagnosis. ${ }^{39-41}$

Multiple risk factors have been studied in an attempt to predict those at higher risk of UUT recurrence post-cystectomy. Pathologic T-stage does not predict for UUT recurrence. ${ }^{40,44}$ 


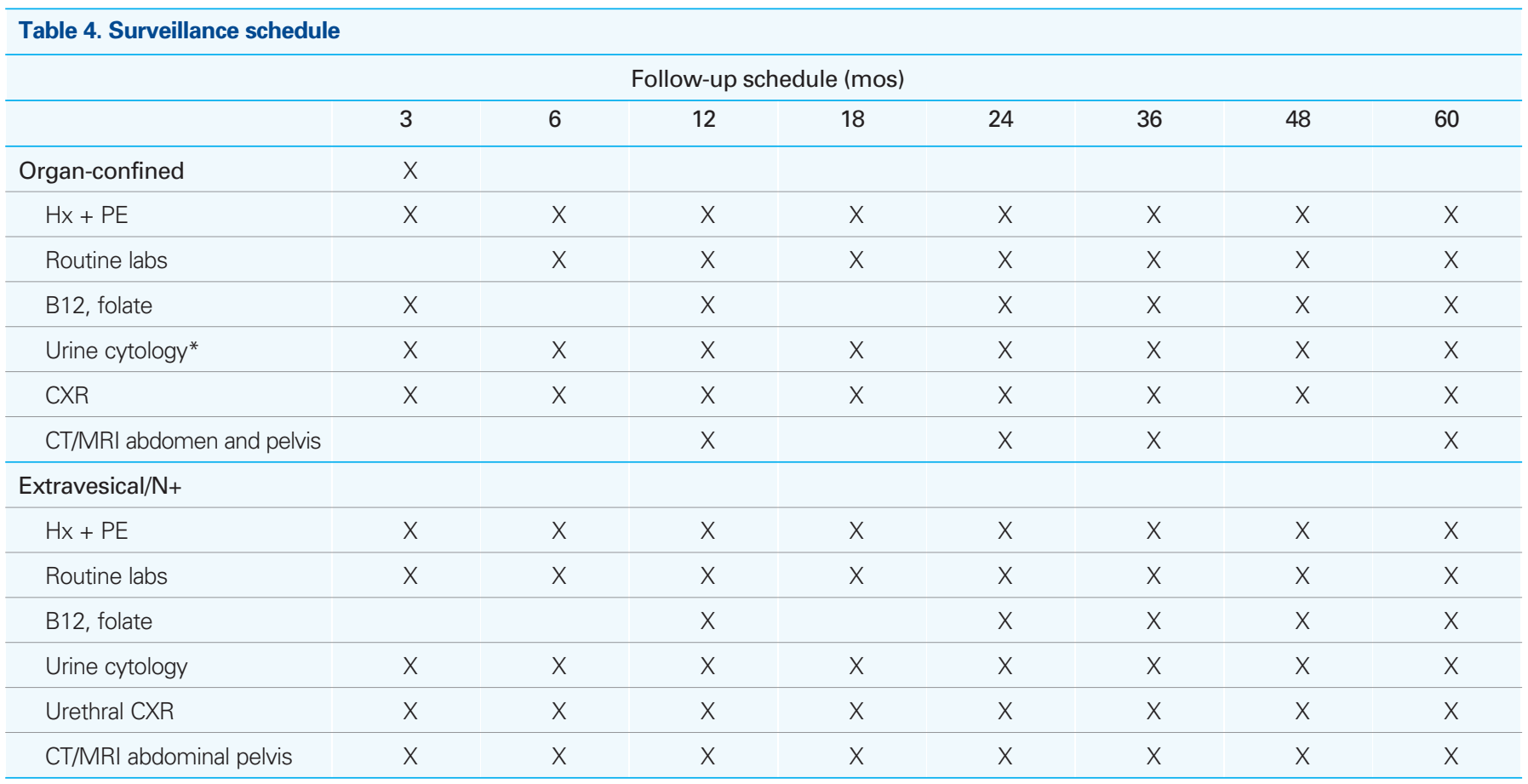

$\mathrm{Hx}=$ history; $\mathrm{PE}=$ physical examination; $\mathrm{CXR}=$ chest $\mathrm{x}$-ray; $\mathrm{CT}=$ computed tomography; $\mathrm{MRI}=$ magnetic resonance imaging; Routine labs = complete blood count, electroloytes, creatinine, calcium, liver function tests; ${ }^{*}=$ bagged cytology in patients with ileoconduits; voided cytology in patients with neobladders; catheter cytology in patients with continent cutaneous reservoirs or hypercontinent neobladders; urethral wash is considered optional only.

Beyond 5 years follow-up should consist of annual $\mathrm{Hx} \& \mathrm{PE}$, routine bloodwork, and cytology with contrast-enhanced CT of the abdomen and pelvis with urography every 2 years.

Tumour involvement of the intramural ureter on final pathology does predict for UUT recurrence, including anastomotic recurrence. Intra-operative positive frozen section analysis of the distal ureteric margin is associated with increased risk of UUT recurrence, however, this is not associated with improved overall survival. ${ }^{45,46}$ Several series show that concomitant carcinoma in situ with muscle invasive disease in the bladder is not associated with UUT recurrence. ${ }^{39-41,44}$ In contrast, in one series of patients undergoing cystectomy for CIS alone there was a higher rate of UUT recurrence than in those with invasive cancer, possibly attributable to a better long-term prognosis and longer period of risk. This longer at-risk interval might increase the risk of UUT recurrence. A large series reviewing risk factors for UUT recurrence found only the presence of associated prostatic urethral involvement to be predictive of subsequent recurrence in the upper tract. ${ }^{42}$ This is to be distinguished from pathologic T4 disease with contiguous prostatic stromal invasion, which was not predictive, probably reflecting reduced survival in these patients and therefore, a shorter at-risk period. ${ }^{44}$ Upper urinary tract recurrences are often advanced at diagnosis and may be associated with a poor prognosis. ${ }^{42}$

\section{Bladder-preserving protocols}

Contemporary bladder-preserving appoaches in patients with clinical stage T2-T4a bladder cancer can achieve 5-year survival rates of $50 \%$ to $60 \%$ and survival rates with an intact bladder of $40 \%$ to $45 \% .{ }^{47}$ The Massachusetts General Hospital has one of the largest experiences with bladder preservation. ${ }^{48}$ Between 1986 and 1997, 190 patients were treated with bladder preservation based on complete response to transurethral resection (TUR) combined with chemotherapy and radiation therapy. The 5- and 10-year disease specific survival was $74 \%$ and $66 \%$ for stage T2 and $53 \%$ and $52 \%$ for stage T3-T4a. The 5 - and 10 -year disease-specific survival with an intact bladder was $46 \%$ and $45 \%$. The pelvic failure rate was $8.4 \%$ With an intact bladder, these patients also continue to be at risk for recurrence of both invasive or non-muscle-invasive bladder cancer. In this series, of the 121 complete responders, 32 (26\%) had non-muscle invasive recurrences. Of these 32 patients, $13(41 \%)$ ultimately required cystectomy. This is similar to a large Ottawa series using intra-arterial cisplatin and radiotherapy for bladder preservation. ${ }^{49}$ In this series, $11 \%$ (16 of 142 with intact bladders) developed non-muscleinvasive bladder cancer recurrence. These tumours can be managed with transurethral resection of bladder tumour 
(TURBT) and intravesical bacillus Calmette-Guérin (BCG) as needed. This risk for subsequent bladder recurrence in the intact bladder necessitates periodic surveillance cystoscopy.

\section{Recommendations}

\section{For radical cystectomy}

The rationale for post-cystectomy surveillance is that earlier detection of recurrence will allow for timely treatment with improved outcomes. This concept has been questioned recently in a retrospective series of 1270 patients with median follow-up of 59 months (range 0 to 270 months) who all underwent routine surveillance testing following cystectomy. ${ }^{18}$ The authors found no difference in survival outcomes whether patients presented with symptoms or had recurrence detected on routine follow-up testing. They hypothesized that symptom-guided follow-up alone may have similar outcomes to surveillance at potentially lower costs. While this hypothesis is provocative, without further confirmation in well-designed prospective trials, it is reasonable for routine surveillance to remain the standard of practice.

The recurrence patterns discussed above indicate that following cystectomy, median time to local and distant recurrence is 12 to 18 months with most patients relapsing within 5 years. Based on the data in Table 3, only $2 \%$ to $3 \%$ of patients relapsed between 5 and 10 years. To maximize the detection of recurrences, surveillance should therefore be intensive for the first 5 years with particular emphasis on the first 2 years. Patients with extravesical and lymph node-positive disease have significantly higher rates of relapse and warrant the most intensive follow-up. Extended pelvic lymphadenectomy to the aortic bifurcation may help reduce local recurrences and improve cancer-specific survival. However, further evidence from randomized trials is needed to demonstrate its utility and to determine whether it will allow for a modified surveillance protocol. Most UUT recurrences occur beyond 3 years with a median of 43 months. The recurrences therefore occur at a time when risk of local and distant recurrence is decreasing. By virtue of their longer survival, patients at lowest risk of local and distant recurrence are paradoxically at greater risk of UUT recurrence. Routine upper tract imaging is therefore advisable in all patients and should continue long term. Additionally, long-term upper tract imaging will allow for the earlier detection of complications of urinary diversion, such as urolithiasis and hydronephrosis secondary to benign stricture.

Routine scheduled surveillance with the goal of identifying local, distant and upper tract recurrences should include clinical assessment along with physical examination. Imaging should consist of contrast-enhanced CT of the abdomen and pelvis including delayed urographic images as well as a chest $\mathrm{x}$-ray for surveillance of pulmonary metastases. In patients with elevated serum creatinine, contrast allergy or other contraindications to CT scanning, MRI is an option. As surveillance of the retained urethra does not appear to improve outcome, urethral wash cytology is optional. Upper tract cytology via bagged urine from an ileoconduit or catherized specimens from continent cutaneous reservoirs as well as voided cytology in patients with neobladders are recommended. Bone and liver involvement can be monitored through serum calcium and liver function tests including alkaline phosphatise levels. It is also reasonable to follow for metabolic complicatons with serum electrolytes, creatinine, vitamins B12 and folate. Recommendations for postcystectomy follow-up are summarized in Table 4.

\section{For bladder-preserving protocols}

As in patients who have undergone cystectomy, the risk of recurrence in patients treated with bladder-sparing appears greatest in the first few years following treatment and diminishes after 5 years. As such, surveillance following bladderpreserving protocols should follow the same schedule outlined in table 4 with patients with clinical stage T2 disease and those with T3-T4a following the organ-confined and extravesical schema, respectively. Additionally, patients should undergo an intial restaging TUR at 3 months following completion of therapy. Surveillance cystoscopy should then be performed at 3-month intervals for the first year, then every 6 months until 5 years, and annually thereafter.

\section{Conclusion}

Patients recurring post-cystectomy for the treatment of urothelial cancer generally have a poor prognosis. Definitive evidence that routine surveillance following cystectomy affects response to treatment and survival is lacking. Nonetheless, it is a generally held oncologic principle that earlier detection of disease at the asymptomatic state positively influences outcome. As such, routine surveillance taking into account a patient's individual risk is justified.

Division of Urology, University of Ottawa, Ottawa, ON

Competing interests: None declared.

This paper has been peer-reviewed. 


\section{References}

1. Canadian Cancer Society/National Cancer Institute of Canada: Canadian Cancer Statistics 2008, Toronto, Canada, 2008

2. Jemal A, Murray T, Ward E, et al. Cancer Statistics 2005. CA Cancer I Clin 2005;55:10-30.

3. Pisters $L$, Westney $L$. The management of locally recurrent invasive bladder cancer following radical cystectomy. Semin Urol Oncol 1996;14:112-9.

4. Bochner BH, Montie JE, Lee CT. Follow-up strategies and management of recurrence in urologic oncology bladder cancer: Invasive bladder cancer. Urol Clin N Am 2003;30:777-89.

5. Whitmore WF, Marshall V. Radical surgery for carcinoma of the urinary bladder. Cancer 1956;9:596-608.

6. Herr, HW. Preoperative irradiation with and without chemotherapy as adjunct to radical cystectomy. Urology 1985;15:127-34.

7. Greven KM, Spira JA, Solin LG, et al. Local recurrence after cystectomy alone for bladder carcinoma. Cancer 1992;69:2767-70.

8. Wishnow KI, Dmochowski R. Pelvic recurrence after radical cystectomy without preoperative radiation. J Urol 1988; 140:42-3.

9. Brendler CB, Steinberg JD, Marshall FF, et al. Local recurrence and survival following nerve-sparing radical cystoprostatectomy. J Urol 1990;144:1137-40.

10. Schoenberg MP, Walsh PC, Breazeale DR, et al. Local Recurrence and survival following nerve sparing radical cystoprostatectomy for bladder cancer: 10-year followup. J Urol 1996; 155:490-4.

11. Stein JP, Lieskovsky $G$, Cote $R$, et al. Radical cystectomy in the treatment of invasive bladder cancer: long-term results in 1054 patients. J Clin Oncol 2001;19:666-75.

12. Skinner DG, Lieskovsky G. Contemporary cystectomy with pelvic node dissection compared to pre-operative radiation therapy plus cystectomy in management of invasive bladder cancer. J Urol 1984;131:1069-72.

13. Roehrborn CG, Sagalowsky Al, Peters PC. Long-term patient survival after cystectomy for regional metastatic transitional cell carcinoma of the bladder. J Urol 1991;146:36-9.

14. Herr HW, Bochner BH, Dalbagni $G$ et al. Impact of the number of lymph nodes retrieved on outcome in patients with muscle invasive bladder cancer. J Urol 2002;167:1295-8.

15. Paulsen AL, Horn T, Steven K. Radical cystectomy, extending the limits of pelvic lymph node dissection improves survival for patients with bladder cancer confined to the bladder wall. J Urol 1998;160:2015-20.

16. Westney OL, Pisters LL, Pettaway CA, et al. Presentation, methods of diagnosis and therapy for pelvic recurrence following radical cystectomy for transitional cell carcinoma of the bladder. J Urol 1998;159:792-5.

17. Tefilli MV, Gheiler EL, Tiguert $R$, et al. Urinary diversion-related outcome in patients with pelvic recurrence after radical cystectomy for bladder cancer. Urology 1999;53:999-1004.

18. Volkmer BG, Kuefer R, Bartsch GC, et al. Oncologic followup after radical cystectomy for bladder cancer - is there benefit? J Urol 2009;181:1587-93.

19. Dalbagni G, Genega E, Hashibe M, et al. Cystectomy for bladder cancer: A contemporary series. J Urol 2001;165:1111-6.

20. Cheng L, Weaver AL, Leibovich BC, et al. Predicting the survival of bladder cancer patients treated with radical cystectomy. Cancer 2000;88:2326-32.

21. Frazier HA, Robertson JE, Dodge RK, et al. The value of pathologic factors in predicting cancer-specific survival among patients treated with radical cystectomy for transitional cell carcinoma of the bladder and prostate. Cancer 1993;71:3993-4001.

22. Saxman SB, Propert KJ, Einhorn LH, et al. Long-term follow-up of a phase III intergroup study of cisplatin alone or in combination with methotrexate, vinblastine and doxorubicin in patients with metastatic urothelial carcinoma: a cooperative group study. J Clin Oncol 1997;15:2564-9.

23. Von der Maase H, Hansen S, Roberts J, et al. Gemcitabine and Cisplatin versus methotrexate, vinblastine, doxorubicin and cisplatin in advanced or metastatic bladder cancer; results of a large, randomized, mutlinationa, multicenter phase III study. J Clin Oncol 2000;17:3068-77.

24. Bajorin DF, Dodd PM, Mazumdar M, et al. Long-term survival in metastatic transitional cell carcinoma and prognostic factor predicting outcome of therapy. I Clin Oncol 1999;17:3173-81.

25. Freeman JA, Esrig D, Stein JP, et al. Management of the patient with bladder cancer. Urethral recurrence. Urol Clin North Am 1994;21:645-51.
26. Freeman JA, Tarter TA, Esrig D, et al. Urethral recurrence in patients with orthotopic ileal neobladders. J Urol 1996;156:1615-9.

27. Lebret $T$, Herve $J M$, Barre $P$, et al. Urethral recurrence of transitional cell carcinoma of the bladder. Predictive value of preoperative latero-montanal biopsies and urethral frozen sections during prostatocystectomy. Eur Urol 1998;33:170-4.

28. Stein JP, Esrig D, Freeman JA, et al. Prospective pathologic analysis of female cystectomy specimens: risk factors for orthotopic diversion in women. Urology 1998;51:951-5.

29. Stein JP, Penson DF, Wu SD, et al. Pathological guidelines for orthotopic urinary diversion in women with bladder cancer: a review of the literature. J Urol 2007;178:756-60.

30. Tobisu K, Tanaka Y, Mizutani T, et al. Transitional cell carcinoma of the urethra in men following cystectomy for bladder cancer: multivariate analysis for risk factors. J Urol 1991;146:1551-3.

31. Levinson AK, Johnson DE, Wishnow KI. Indications for urethrectomy in an era of continent urinary diversion. J Urol 1990;144:73-5.

32. Zabbo A, Montie JE. Management of the urethra in men undergoing radical cystectomy for bladder cancer. J Urol 1984;131:267-8.

33. Clark PE, Stein JP, Groshen SG, et al. The management of urethral transitional cell carcinoma after radical cystectomy for invasive bladder cancer. J Urol 2004;172(4Pt1):1342-7.

34. Poole-Wilson DS, Barnard RJ. Total cystectomy for bladder tumours. Br J Urol 1971;43:16-24.

35. Baron JC, Gibod LB, Steg A. Management of the urethra in patients undergoing radical cystectomy for bladder carcinoma. Eur Urol 1989;16:283-5.

36. Lin DW, Herr HW, Dalbagni G. Value of urethral wash cytology in the retained male urethra after radical cystoprostatectomy. J Urol 2003;169:961-3.

37. Balaii KC, McGuire M, Grotas J, et al. Upper tract recurrences following radical cystectomy. An analysis of prognostic factors, recurrence pattern and stage at presentation. J Urol 1999;162:1603-6.

38. Huguet-Perez J, Palou J, Millan-Rodriguez F, et al. Upper tract transitional cell carcinoma following cystectomy for bladder cancer. Eur Urol 2001; 40:318-23.

39. Tsuji Y, Nakamura H, Ariyoshi A. Upper tract involvement after cystectomy and ileal conduit diversion for primary bladder carcinoma. Eur Urol 1996:29:216-20.

40. Kenworthy $P$, Tanguay S, Dinney CPN. The risk of upper tract recurrence following cystectomy in patients with transitional cell carcinoma involving the distal ureter. J Urol 1996;155:501-3.

41. Tran W, Serio AM, Raj GV, et al. Longitudinal risk of upper tract recurrence following radical cystectomy for urothelial cancer and the potential implications for long-term surveillance. J Urol 2008;179:96-100.

42. Sved PD, Gomez P, Nieder AM, et al. Upper tract tumour after radical cystectomy for transitional cell carcinoma of the bladder: incidence and risk factors. BJU Int 2004;94:785-9.

43. Akkad $T$, Gozzi C, Deibl $M$, et al. Tumour recurrence in the remnant urothelium of females undergoing radical cystectomy for transitional cell carcinoma of the bladder: long-term results from a single center. J Urol 2006;175:1268-71.

44. Sanderson KM, Stein JP, Cai J, et al. Upper tract recurrence following radical cystectomy for transitional cell carcinoma. an analysis of 1069 patients with 10-year follow-up. J Urol 2007;177:2088-94.

45. Yossepowitch 0 , Dalbagni $G$, Golijanin D, et al. Orthotopic urinary diversion after cystectomy for bladder cancer: implications for cancer control and patterns of disease recurrence. J Urol 2003;169:177-81.

46. Sanderson KM, Rouprêt M. Upper urinary tract tumour after radical cystectomy for transitional cell carcinoma of the bladder: an update on the risk factors, surveillance regimens and treatments. BJU Int 2007;100:11-6.

47. Shipley WU, Kaufman DS, Zehr E, et al. Selective bladder preservation by combined modality protocol treatment: long-term outcomes of 190 patients with invasive bladder cancer. Urology 2002;60:62-7.

48. Mak RH, Zietman AL, Heney NM, et al. Bladder preservation: optimizing radiotherapy and integrated treatment strategies. BJU Int 2008;102:1345-53.

49. Eapen L, Stewart D, Collins J, et al. Effective bladder sparing therapy with intra-arterial cisplatin and radiotherapy for localized bladder cancer. J Urol 2004:172:1276-80.

Correspondence: Dr. Christopher Morash, The Ottawa Hospital Prostate Cancer Assessment Centre Civic Campus Office, 1053 Carling Avenue, Ottawa, ON KIY 4E9; fax: 613-761-5305; cmorash@ottawahospital.on.ca 